\title{
DERECHO Y POLÍTICAS AMBIENTALES EN LA REGIÓN DE MURCIA (PRIMER SEMESTRE 2018)
}

\author{
ELISA PÉrez de los Cobos HERnÁndeZ \\ Profesora Ayudante Doctor de Derecho Administrativo \\ Universidad de Murcia \\ SANTIAGO M. ÁlVAREZ CARREÑo \\ Profesor Titular de Derecho Administrativo \\ Universidad de Murcia
}


Sumario: 1. Breve reflexión introductoria. 2. Manifestaciones de la política y legislación ambiental de la Administración regional en Murcia. 2.1. Órganos Directivos de la Consejería de Turismo, Cultura y Medio Ambiente: Dirección General del Mar Menor.2.2. Protección y defensa de los animales de compañía de la Región de Murcia. 2.3. Mar Menor: Ley de medidas urgentes para garantizar la sostenibilidad ambiental en el entorno del Mar Menor y otras actuaciones de las Administraciones públicas: A. Ley de Medidas urgentes para garantizar la sostenibilidad ambiental en el entorno del Mar Menor. B. Proyecto sobre la Monitorización y Modelado de la Calidad de Aguas y Estado Ecológico del Mar Menor y Prevención de Impactos: modificación del convenio para la concesión directa de la subvención a la Universidad de Murcia. 2.4. Urbanismo: Instrucciones interpretativas. 2.5. Residuos: A. Recogida de residuos en el mar. B. Residuos sólidos. 2.6. Plan de Mejora de la Calidad del Aire para la Región de Murcia 2016-2018. 2.7. Programa de Medio Ambiente y Acción por el Clima (LIFE). 2.8. Premios de desarrollo sostenible. 2.9. Agricultura: A. Medidas fitosanitarias. B. Agricultura ecológica y denominación de origen. C. Programa de Desarrollo Rural de la Región de Murcia 2014-2020: ayudas para las medidas de "agroambiente y clima" y "agricultura ecológica". D. Programa de Desarrollo Rural de la Región de Murcia 2014-2020: Ayudas LEADER. 2.10. Pesca: A. Veda temporal para el ejercicio de la pesca de cerco en aguas interiores de la Región de Murcia. B. Reserva Marina zona de cabo de Palos-Islas Hormigas. 3. Ordenanzas locales. 3.1. Murcia: A. Aprobación inicial de la ordenanza municipal sobre protección y tenencia de animales de convivencia humana. B. Aprobación inicial del Plan Especial de Ordenación y Protección de la Zona Arqueológica del Arrabal de la Arrixaca Nueva. 3.2 Caravaca de la Cruz: Aprobación inicial de la ordenanza municipal para la protección y uso sostenible del paraje de Las Fuentes del Marqués. 3.3. Molina de Segura: Aprobación inicial de la modificación de la ordenanza municipal para la tenencia, protección y bienestar de los animales. 3.4. Los Alcázares: Reglamento de autorización del procedimiento de otorgamiento de autorizaciones de dominio público y del régimen jurídico de los Huertos Urbanos del Ayuntamiento de Los Alcázares.

\section{BREVE REFLEXIÓN INTRODUCTORIA}

La presente crónica trata de reflejar la producción normativa y la aprobación de instrumentos de carácter ambiental en la Comunidad Autónoma de la Región de Murcia durante este primer semestre del 2018. Como nuestro fiel lector podrá fácilmente detectar determinadas cuestiones, señaladamente la destrucción progresiva del equilibrio ambiental de la laguna costera del Mar Menor, ocupan una parte central de las referencias, como si una precipitada aprobación de normas y planes pudiera venir a solucionar por arte de magia los graves problemas generados durante décadas de abandono y de feliz ignorancia.

Además de otras cuestiones que se repiten cíclicamente (las modificaciones organizativas, las órdenes de veda para la pesca y caza, las subvenciones de determinados proyectos, los premios ambientales y otras) en la presente crónica recuperamos la exposición de ordenanzas ambientales municipales que se han aprobado durante el periodo de referencia. 
Un tratamiento singular queda reservado a la flamante Ley de Protección y Defensa de los animales de compañía que esperemos contribuya a modificar los comportamientos crueles que, por desgracia, todavía son muy frecuentes en el trato de "nuestros seres queridos", en feliz expresión del inefable EVELYN WAUGH.

\section{MANIFESTACIONES DE LA POLÍTICA Y LEGISLACIÓN AMBIENTAL DE} LA ADMINISTRACIÓN REGIONAL EN MURCIA

\section{1. Órganos Directivos de la Consejería de Turismo, Cultura y Medio Ambiente: la Dirección General del Mar Menor}

El Mar Menor es una de las mayores lagunas litorales de Europa y la más grande de la Península Ibérica. Sus singulares valores ambientales determinaron su incorporación a los Humedales de Importancia Internacional (RAMSAR) y Zonas Especialmente Protegidas de Importancia para el Mediterráneo (ZEPIM), así como la declaración del Paisaje Protegido de los Espacios Abiertos e Islas del Mar Menor, del Parque Regional de Salinas y Arenales de San Pedro del Pinatar, del Lugar de Importancia Comunitaria (LIC) "Mar Menor" y de la Zona de Especial Protección para las Aves (ZEPA) "Mar Menor". En este emblemático lugar convergen múltiples usos y aprovechamientos, principalmente turísticos, recreativos, salineros y pesqueros, con un importante aprovechamiento agrícola de su entorno que convierten la laguna en un factor clave en los planes de desarrollo regional. De ahí que de la problemática ambiental del Mar Menor se estén derivando gravísimas consecuencias a nivel económico y social que no pueden ser ignoradas debiendo integrarse como un todo en la consideración global del problema.

Para el logro de soluciones realmente eficaces, resulta imprescindible el Decreto 266/2017, de 29 de noviembre, por el que se modifica el Decreto de Consejo de Gobierno 75/2017, de 17 de mayo, por el que se establecen los Órganos Directivos de la Consejería de Turismo, Cultura y Medio Ambiente 1. Este Decreto atribuye a favor de la Dirección General del Mar Menor, creada en

\footnotetext{
${ }^{1}$ BORM núm. 281, de 5 de diciembre de 2017.
} 
2017, las competencias y funciones de ejecución de los proyectos y actuaciones relacionados con la protección y regeneración ambiental de su ecosistema, sin perjuicio de las atribuidas a otros centros directivos de la Administración Regional $^{2}$; reconoce a favor de la Dirección General del Mar Menor la coordinación con los distintos organismos y direcciones generales de la Comunidad Autónoma, y con otras Administraciones Publicas y entidades públicas o privadas con competencias concurrentes para el desarrollo de dichos proyectos y actuaciones, y el impulso del conocimiento científico y la investigación aplicada en relación con problemas ambientales de la laguna. Por último, adscribe a la Dirección General del Mar Menor tanto el Comité de Asesoramiento Científico del Mar Menor como el Comité de Participación Social del Mar Menor.

El Comité de asesoramiento científico del Mar Menor, constituido el día 15 de septiembre de 2016, se configura como un grupo de trabajo, perteneciente a la Consejería de Agua, Agricultura y Medio Ambiente ${ }^{3}$. Sus funciones abarcan no sólo el asesoramiento científico en la selección y ejecución de acciones dirigidas a la mejora del estado ecológico del Mar Menor, sino el impulso del conocimiento científico y la investigación aplicada en relación con problemas ambientales de la laguna. Desde su puesta en marcha, el Comité ha resaltado la necesidad de profundizar en el conocimiento de los actores que afectaban al estado del ecosistema teniendo en cuenta la rapidez con la que estaban

\footnotetext{
${ }^{2}$ En este sentido vid. nuestra crónica anterior en Revista Catalana de Dret Ambiental, Vol. VIII, Núm. 2, 2017, p. 1-20.

En especial, el análisis realizado sobre el Decreto de la Presidencia de la Comunidad Autónoma 3/2017, de 4 de mayo, de reorganización de la Administración regional, por el que se modificó la denominación y las competencias de las distintas Consejerías, efectuando una nueva distribución competencial entre los departamentos de la Administración Regional. Y de otro lado, el Decreto de Consejo de Gobierno 75/2017, de 17 de mayo, modificado posteriormente por el Decreto 223/2017, de 2 de agosto, por el que se establecieron los Órganos Directivos de la Consejería de Turismo, Cultura y Medio Ambiente, y, en cuya virtud, se creó la Dirección General del Mar Menor.

Esta Dirección General, como señala el art. 7 del Decreto, asume las competencias y funciones de impulso, coordinación y seguimiento de los planes, proyectos y actuaciones en el Mar Menor, especialmente aquellos relacionados con la protección y regeneración ambiental y natural del ecosistema del Mar Menor, promovidos por la Administración Regional o mediante otras fórmulas con participación de la Administración regional, así como la coordinación con los distintos organismos y centros directivos de la Comunidad Autónoma de la Región de Murcia y con otras Administraciones Públicas o entidades públicas o privadas con competencias en el desarrollo de los mismos.

${ }^{3}$ Dicha orden fue modificada por la Orden, de 30 de diciembre de 2016 de la Consejería de Agua, Agricultura y Medio Ambiente para incorporar a los mismos funcionarios expertos provenientes de distintas consejerías relacionadas con la problemática ambiental del Mar Menor (BORM, núm. 3, de 5 de enero de 2017).
} 
evolucionando algunos parámetros físicos, químicos y biológicos, y sobre todo su incidencia sobre los sedimentos y comunidades bentónicas. De ahí que los primeros esfuerzos se materializasen en la promoción de proyectos y campañas para paliar el déficit de conocimiento y garantizar un seguimiento y monitorización adecuados de los distintos elementos de la laguna.

La modificación del Decreto 75/2017, de 17 de mayo, obliga a su vez a variar la composición original del Comité de asesoramiento científico del Mar Menor. Se da una nueva redacción al artículo 3 de la Orden, de 29 de julio de 2016 de la Consejería de Agua, Agricultura y Medio Ambiente, por la que se crea el Comité de Asesoramiento Científico del Mar Menor, de acuerdo con el cual estará integrado por el Presidente, que será el Director General del Mar Menor; por un miembro de cada uno de los grupos de investigación con experiencia en las líneas de investigación sobre los problemas ambientales del Mar Menor pertenecientes a las Universidades de la Región de Murcia, el Instituto Murciano de Investigación y Desarrollo Agrario y Alimentario y el Instituto Español de Oceanografía, elegido por cada grupo; así como por un funcionario de cada uno de los siguientes centros directivos: Dirección General del Mar Menor, Dirección General de Medio Natural, Dirección General de Medio Ambiente, Dirección General del Agua y Dirección General de Agricultura, Ganadería, Pesca y Acuicultura, designado por sus respectivos titulares. Actuará como secretario el director de la Oficina de Impulso Socioeconómico del Medio Ambiente ${ }^{4}$.

\subsection{Protección y defensa de los animales de compañía de la Región de Murcia}

El año 2017 cierra con la entrada en vigor, el 23 de diciembre, de la Ley $6 / 2017$, de 8 de noviembre, de protección y defensa de los animales de compañía de la Región de Murcia ${ }^{5}$. Una Ley que, apostando por el sacrificio cero y la plena intolerancia frente al abandono y al maltrato, sitúa a la Región de Murcia a la vanguardia del bienestar animal. Su fundamento, según se

\footnotetext{
${ }^{4}$ Orden de la Consejería de Turismo, Cultura y Medio Ambiente, por la que se modifica la Orden, de 29 de julio de 2016, de la Consejería de Agua, Agricultura y Medio Ambiente, por la que se crea el Comité de Asesoramiento Científico del Mar Menor (BORM, núm. 4, de 5 de enero de 2018).

${ }^{5}$ BORM, núm. 271, de 23 de noviembre de 2017.
} 
manifiesta en el propio preámbulo de la norma, responde a «la necesidad de promulgar un instrumento jurídico más eficaz en la defensa y protección de los animales de compañía, cuya finalidad esencial es profundizar en las medidas educativas y de concienciación social de la población, pero también de endurecimiento del régimen sancionador y ello con la finalidad de erradicar esos comportamientos de maltrato y de abandono». Se corrigen así las carencias de la ya derogada Ley 10/1990, de 27 de agosto, de protección y defensa de animales de compañía de la Región de Murcia (a excepción del art. 9, que conserva su vigencia con rango reglamentario), que tras más de tres décadas sin desarrollo reglamentario se mostraba ineficaz para atajar con firmeza conductas incívicas y crueles con los animales de compañía demasiado frecuentes en nuestra sociedad. Se debe anotar, sin embargo, que la norma no incluye en su marco de regulación a todas las especies animales, limitándose a los animales de compañía, dejando en el tintero, además, cuestiones tan esenciales como la existencia de un registro de maltratadores que permita incrementar la seguridad jurídica. En cualquier caso, esta Ley constituye un importante avance hacia un marco normativo de protección integral hacia los animales en coherencia con las demandas sociales.

Entre las novedades de mayor implicación práctica puede destacarse que la Ley establece una prohibición general de mantener atados a los animales de compañía en el entorno domiciliario. El uso incorrecto de ataduras puede producir en los animales graves lesiones físicas y psíquicas motivo por el que la norma fija unos parámetros de obligado cumplimiento que garanticen el bienestar cuando, en momentos puntuales, los ciudadanos deban atar a sus animales. En todo caso, esta situación temporal no podrá superar las diez horas continuadas al día (art 7.3).

En segundo lugar, se crea un Registro de Animales de Compañía de la Región de Murcia, adscrito a la consejería competente en materia de protección y sanidad animal (art. 15). El objetivo es garantizar un control y seguimiento sanitario más adecuado de estos animales a lo largo de su vida con independencia del municipio en que residan o de los cambios de propietario.

Asimismo, se apuesta por la protección y el control poblacional de las colonias felinas. Los gatos callejeros forman parte de la fauna urbana de las ciudades y 
están integrados en el medio ambiente en el que desarrollamos nuestra vida cotidiana, si bien, en caso de superpoblación pueden generarse problemas medioambientales o sanitarios. La Ley establece un procedimiento de identificación y cuidado para las colonias felinas callejeras atribuyendo a los Ayuntamientos la vigilancia sanitaria y el control de estas poblaciones. $Y$ se contempla una alternativa a la captura y sacrificio eutanásico de los gatos callejeros a través de un método que prevé que, tras su captura y control sanitarios, sean identificados, esterilizados y devueltos a la colonia (art. 25).

La norma impulsa acciones divulgativas y de formación promoviendo el cuidado y tenencia responsable de los animales domésticos. Asimismo, se fomentan las adopciones y programas de concienciación y sensibilización sobre abandono de animales. Para ello se apoya en una mejor coordinación y colaboración interadministrativa que parte de la correcta clarificación del ámbito de actuación de cada Administración Pública implicada. La Ley individualiza las competencias municipales y las competencias de la administración regional, incluyéndose, además, una distribución competencial de las consejerías y órganos directivos con competencias en materia de protección y sanidad animal, salud pública y medio ambiente. Se apuesta por lo tanto por identificar espacios competenciales de actuación independientes y, en aquellos casos en que ello no sea posible, por garantizar mecanismos de coordinación que posibiliten una actuación conjunta ágil y eficaz.

La Ley acrecienta la actuación inspectora y endurece el régimen de infracciones y sanciones aplicables. Se incluye una extensa tipificación con un listado amplio y exhaustivo de infracciones, leves, graves o muy graves, a las que se apareja un endurecido régimen de sanciones pecuniarias, pasando la sanción mínima de $30 €$ a $100 €$ y la máxima de $3.000 €$ a $30.000 €$. Además, su disposición adicional primera atribuye carácter finalista a las cuantías obtenidas por las sanciones impuestas que serán destinadas al fomento y protección de estos animales.

Una de las más significativas novedades está en la prohibición del sacrificio de animales, esto es, la medida de "sacrificio cero". El sacrificio, definido en la Ley como la muerte provocada a un animal, sin que se lleve a cabo para evitarle un sufrimiento o por razones de sanidad animal, de salud pública, de seguridad o 
medioambientales queda taxativamente prohibido. La norma solo contempla los casos de eutanasia limitando la realización de la misma a unos supuestos tasados, como la evitación de sufrimiento del animal o por motivos de sanidad y seguridad de las personas o animales o de existencia de riesgo para la salud pública o el medio ambiente, y bajo unos controles y requisitos clínicos específicos como su prescripción y ejecución por un veterinario, de forma rápida e indolora, previa sedación y mediante métodos que impliquen el mínimo sufrimiento y provoquen una pérdida de consciencia inmediata.

Por último, y como se avanzaba al principio, la nueva Ley de protección y defensa de los animales de compañía de la Región de Murcia presenta luces y sombras. Así, aun cuando se prohíbe expresamente la instalación y los espectáculos de circos con animales silvestres, estos quedan fuera del ámbito de protección de la Ley. Al limitar su aplicación a la defensa de los animales de compañía, la norma deja fuera muchos de las reclamaciones que, desde las asociaciones protectoras y otras entidades dedicadas al bienestar de los animales, se llevan haciendo desde hace décadas. No obstante, debe reconocerse que es un paso al frente en la protección integral de todas las especies animales que debe ser el objetivo último a cumplir.

\subsection{Mar Menor: Ley de medidas urgentes para garantizar la sostenibilidad ambiental en el entorno del Mar Menor y otras actuaciones de las Administraciones públicas}

A. Ley de Medidas urgentes para garantizar la sostenibilidad ambiental en el entorno del Mar Menor

Pese a los irreemplazables valores ambientales del Mar Menor y su fundamental importancia estratégica y económica para nuestra Región, la laguna ha sufrido un proceso de transformación y detrimento imparable. Una de las consecuencias más evidentes es el notable deterioro de la calidad de sus aguas que ha conducido a la progresiva eutrofización de la laguna.

La gravedad del problema hace que no exista una solución única y sencilla. Debe hallarse una respuesta válida para el total de variables económicas, sociales y ambientales que allí concurren, cada una de las cuales presenta una 
singular complejidad técnica. Esta solución deberá ser el resultado de la combinación de diversas actuaciones en los diferentes sectores de actividad que han convergido para que en estos momentos el Mar Menor se encuentre en la deplorable situación supra señalada.

Aun cuando son muchos los factores que concurren en la problemática descrita, el "Informe integral sobre el estado ecológico del Mar Menor", de 13 de febrero de 2017, elaborado por el Comité de Asesoramiento Científico del Mar Menor, identifica la contaminación por nitratos y compuestos orgánicos persistentes, como factores que han contribuido al desequilibrio ambiental de la laguna, sin minusvalorar la contaminación por metales pesados o la procedente de aguas de escorrentías. Se aprueba entonces el Decreto-Ley 1/2017, de 4 de abril, de medidas urgentes para garantizar la sostenibilidad ambiental en el entorno del Mar Menor. Si bien, aun cuando una considerable parte del Campo de Cartagena ya ha sido declarada como zona vulnerable a la contaminación por nitratos, siéndole de aplicación el programa de actuación aprobado por la Consejería de Agua, Agricultura y Medio Ambiente, se ha considerado necesario y urgente intensificar las acciones de protección, procurando una mayor sostenibilidad ambiental de las actividades que se realizan en el entorno del Mar Menor 6 .

En este contexto de preocupación por la imparable degradación del Mar Menor se aprueba la Ley $1 / 2018$, de 7 de febrero, de medidas urgentes para garantizar la sostenibilidad ambiental en el entorno del Mar Menor que entró en vigor el pasado 14 de febrero. Esta norma se circunscribe a la adopción de medidas urgentes para la ordenación y sostenibilidad de las actividades agrarias en el entorno del Mar Menor, intentando garantizar su aplicación y protección de los recursos naturales de este espacio, "mediante la eliminación o reducción de las afecciones provocadas por vertidos, arrastres de sedimentos

\footnotetext{
${ }^{6}$ En este sentido, cabe señalar que la Directiva 91/676/CEE, de 12 de diciembre, relativa a la protección de las aguas contra la contaminación producida por nitratos de origen agrario, traspuesta al ordenamiento jurídico español mediante el Real Decreto 26/1996, de 16 de febrero, impone a los estados miembros la designación de zonas vulnerables a la contaminación por nitratos de origen agrario, la elaboración de un Código de Buenas Prácticas Agrarias y la confección de programas de actuación.
} 
y cualesquiera otros elementos que puedan contener contaminantes perjudiciales para la recuperación de su estado ecológico"7.

La Ley, integrada por 26 artículos, se divide en cinco capítulos, que se completan con cinco disposiciones adicionales, una disposición transitoria, una disposición derogatoria y tres disposiciones finales. El capítulo I identifica tanto su objeto como su ámbito de aplicación que abarca, además de la laguna costera, los términos municipales de San Pedro del Pinatar, San Javier, Los Alcázares, Torre Pacheco, Fuente Álamo, Cartagena, La Unión y Murcia que integran la cuenca hidrográfica vertiente. El capítulo II prevé las diferentes medidas de sostenibilidad ambiental aplicables a las explotaciones agrarias incluidas en las tres zonas en las que se ha dividido el Campo de Cartagena ${ }^{8}$. La finalidad que se pretende es la de preservar los recursos naturales y valores ambientales del Mar Menor, y en especial, los hábitats que han dado lugar a la designación del LIC “Mar Menor" y de la ZEPA “Mar Menor". Entre las medidas que se contemplan se encuentra la obligación de implantación de estructuras vegetales de barrera y conservación, requisitos para el laboreo del suelo, limitaciones de las actividades agrícolas en terrenos próximos al dominio público marítimo terrestre, que incluye la prohibición de aplicación de fertilizantes en la zona de servidumbre de protección, prohibiendo la existencia de cultivos, excepción hecha de invernaderos y leñosos ya implantados. Junto con estas se contemplan otras medidas, todas ellas concernientes a la práctica de la agricultura en el espacio afectado por la Ley, como la prohibición de apilamiento temporal de estiércol.

El capítulo III se centra en el control de los vertidos al Mar Menor prohibiéndolos de forma general; solo se contempla una excepción respecto de los vertidos de aguas pluviales que serán posibles en aquellos casos en que no resulte viable su eliminación por otros medios, debiendo los ayuntamientos realizar las inversiones encaminadas al cumplimiento de este objetivo, con el apoyo financiero de la Comunidad Autónoma que contempla la disposición

\footnotetext{
${ }^{7}$ Ley $1 / 2018$, de 7 de febrero, de medidas urgentes para garantizar la sostenibilidad ambiental en el entorno del Mar Menor (BORM, núm. 36, de 13 de febrero de 2018).

${ }^{8}$ La zona 1 comprende las explotaciones agrícolas situadas en la franja más próxima al Mar Menor. La zona 2 abarca la zona vulnerable correspondiente a los acuíferos Cuaternario y Plioceno en el área definida por zona regable oriental del Trasvase Tajo-Segura y litoral del Mar Menor en el Campo de Cartagena. La zona 3 se extiende por el resto de la cuenca vertiente.
} 
adicional tercera. Para la tramitación preferente y declaración de urgencia de las actuaciones relacionadas con los fines de la norma, el capítulo IV prevé diversas medidas, por ejemplo, la reducción de los plazos a la mitad de los establecidos en el procedimiento ordinario, que también se orientan a clarificar los supuestos en que es exigible el trámite de evaluación ambiental en la actividad agrícola, y a facilitar la expropiación forzosa de los bienes y derechos que puedan quedar afectados por las inversiones necesarias. La efectividad de todas estas limitaciones se asegura mediante el régimen sancionador y de control que desarrolla el capítulo $\mathrm{V}$ y en el que se identifican los órganos competentes, la función de control, los sujetos responsables, el régimen de infracciones leves, graves y muy graves y sus correspondientes sanciones cuyo máximo alcanza los $100.000 €$.

Finalmente, esta norma contiene dos importantes medidas, de especial importancia en las prácticas agrarias del Campo de Cartagena y que constituyen un complemento necesario para el cumplimiento de sus objetivos, pero que por ser de aplicación a todo el ámbito territorial regional aparecen como disposiciones adicionales: la aprobación de un nuevo Código de Buenas Prácticas Agrarias de la Región de Murcia, que se inserta en el Anexo V (disposición adicional primera); y el establecimiento de un régimen sancionador específico por incumplimientos de la normativa de protección de las aguas frente a la contaminación por nitratos de fuentes agrarias (disposición adicional segunda).

B. Proyecto sobre la Monitorización y Modelado de la Calidad de Aguas y Estado Ecológico del Mar Menor y Prevención de Impactos: modificación del convenio para la concesión directa de la subvención a la Universidad de Murcia

Desde las distintas Administraciones se trabaja con intensidad con el fin conocer en profundidad las causas y la evolución de la situación ambiental del Mar Menor, destacando diversas inversiones en proyectos de seguimiento e investigación con las Universidades de Murcia y Politécnica de Cartagena, el Instituto Español de Oceanografía, el Instituto Geológico y Minero de España, el CEBAS-CSIC y el IMIDA, así como de las experiencias prácticas de algunas organizaciones de conservación y defensa de la naturaleza, entre otros. 
Mediante Decreto 122/2016, de 26 de octubre, el Consejo de Gobierno aprobó las normas reguladoras de la concesión directa de una subvención a la Universidad de Murcia para el desarrollo del proyecto sobre la monitorización y modelado de la calidad de las aguas y estado ecológico del Mar Menor por importe de 244.000,00 $€^{9}$. Con fecha 13 de diciembre de 2016 se firmó el Convenio de Colaboración entre la Comunidad Autónoma de la Región de Murcia, a través de la Consejería de Agua, Agricultura y Medio Ambiente y la Universidad de Murcia para instrumentar la concesión de la subvención. Según la Memoria que figura como Anexo I del Convenio, entre los objetivos específicos del Proyecto los contemplados bajo los números 4, 5 y 6 implican el desarrollo y aplicación de modelos numéricos, que precisan la utilización del modelo hidrodinámico SHYFEM, desarrollado en el ISMAR-CNR (Institute of Marine Science, National Research Council) de Venecia, siendo imprescindible para el cumplimiento de los citados objetivos contar con la colaboración de esta entidad en la ejecución parcial de la actividad.

Teniendo en cuenta que en el Convenio suscrito no se prevé de forma expresa la posibilidad de subcontratar, siendo esto preciso para la ejecución parcial de la actividad, se considera necesario modificar el citado Convenio incluyendo una nueva cláusula en la que se prevea la posibilidad de subcontratar de conformidad con lo dispuesto en el artículo 24 de la Ley 7/2005, de 18 de noviembre, de Subvenciones de la Comunidad Autónoma de la Región de Murcia y en los términos establecidos en el artículo 29 de la Ley 38/2003, de 17 de noviembre, General de Subvenciones. Esta nueva previsión no conlleva la modificación del importe global presupuestado, pero sí la modificación del Anexo II (Presupuesto) del Convenio en lo que se refiere al desglose de los gastos de ejecución.

Igualmente se han ampliado los plazos de ejecución y justificación de la actividad subvencionada lo que supone una modificación del plazo de vigencia del Convenio que se prolonga hasta el 17 de agosto de 2018.

Por Orden de la Consejería de Turismo, Cultura y Medio Ambiente se aprueba la modificación del texto del Convenio de colaboración entre la Comunidad

\footnotetext{
${ }^{9}$ BORM núm. 259, de 8 de noviembre de 2016.
} 
Autónoma de la Región de Murcia, a través de la Consejería de Turismo, Cultura y Medio Ambiente, y la Universidad de Murcia para el desarrollo del proyecto sobre la monitorización y modelado de la calidad de aguas y estado ecológico del mar menor y prevención de impactos suscrito con fecha 13 de diciembre de $2016^{10}$.

\subsection{Urbanismo: Instrucciones interpretativas}

Ley $13 / 2015$, de 30 de marzo, de ordenación territorial y urbanística de la Región de Murcia (en adelante, LOTURM), introdujo diversas novedades cuya puesta en práctica ha motivado la necesidad de recurrir a distintas Instrucciones interpretativas por parte de la Consejería de Presidencia y Fomento ${ }^{11}$.

Así, la LOTURM introdujo ex novo el régimen de fuera de ordenación, incorporando, como novedad, el régimen de fuera de norma. Este régimen se aplica a edificaciones que incumplen alguna condición normativa pero no son sustancialmente disconformes con el plan, admitiéndose usos y obras que no agraven la situación de disconformidad normativa, con el fin de regularizar situaciones muy frecuentes en la realidad y posibilitar el mantenimiento de actividades y usos preexistentes y no declarados ilegales. Si bien, ha sido necesario recurrir a una Instrucción interpretativa por parte de la Consejería de Presidencia y Fomento sobre la situación de fuera de norma prevista en el artículo 112 LOTURM $^{12}$, a efectos de esclarecer cuestiones tales como la propia definición y circunstancias para que una edificación sea considerada en situación de fuera de norma; el concepto de "sustancialmente disconforme" con el planeamiento; o cuestiones específicas relativas al fuera de norma como el plazo para la restitución de la legalidad urbanística.

\footnotetext{
${ }^{10}$ BORM, núm. 260, 10 de noviembre de 2017.

${ }^{11}$ La LOTURM faculta en su disposición adicional tercera al consejero competente en materia de ordenación territorial o en materia de urbanismo para dictar instrucciones interpretativas de las disposiciones normativas e instrumentos relacionados con la materia, previo informe de los órganos consultivos y de participación previstos en la Ley.

${ }^{12}$ Orden, del 13 de diciembre de 2017, de la Consejería de Presidencia y Fomento, de aprobación de la Instrucción sobre la situación de fuera de norma prevista en el artículo 112 de la Ley 13/2015, de 30 de marzo, de Ordenación Territorial y Urbanística de la Región de Murcia (BORM, núm. 289, de 16 de diciembre de 2017).
} 
Las conclusiones que pueden extraerse de los instrumentos normativos señalados son las siguientes:

Primero.- Tanto las instalaciones, como las construcciones y edificaciones son susceptibles de encontrarse en situación de fuera de norma.

Segundo.- El concepto de "sustancialmente disconforme con el planeamiento" ha de fijarse técnicamente, caso a caso, disponiendo el técnico informante de criterios en la propia ley que pueden orientar la definición de este concepto, en el supuesto de que no se encuentren establecidos en el propio Plan, y entender esta situación como aquella que afecta a edificaciones o instalaciones que no impiden la ejecución de las determinaciones del planeamiento.

Tercero.- El plazo para iniciar un expediente de restitución de la legalidad urbanística es de 4 años desde que se concluyeron las obras.

Cuarto.- La situación de fuera de norma se puede dar tanto por incumplimientos normativos de planeamiento urbanístico como de la planificación territorial.

Quinto.- No existe una "declaración" de fuera de norma como acto separado del título urbanístico o resolución administrativa en que se autoriza una edificación.

Sexto.- El que un inmueble se encuentre en situación de fuera de norma no impide la autorización del uso excepcional ni la licencia o título habilitante que corresponda, siempre que con ello no se agrave el aspecto normativo que determinó su disconformidad.

Séptimo.- No se puede paralizar un expediente de restitución de la legalidad urbanística por el hecho de encontrarse en situación de fuera de norma, salvo lo previsto excepcionalmente en el artículo 279 de la ley, referido a la legalización de actuaciones con disconformidades no sustanciales de la normativa urbanística aplicable.

Además de lo anterior debe tenerse en cuenta que la legislación urbanística siempre ha dedicado una especial atención al régimen urbanístico del suelo en relación con las distintas clases y categorías establecidas por el planeamiento, determinando sus posibilidades de edificación y uso, tanto en los ámbitos 
ordenados y planificados como en aquellos que no son susceptibles de transformación urbanística (suelo no urbanizable) o los que no son objeto de previsión de desarrollo (urbanizable sin sectorizar) que quedan por tanto al margen del proceso "reglado" de la planificación y la gestión urbanística. Es en estos casos, donde además de los usos propios del suelo permitidos en situación rural por estar ligados a la actividad productiva, se plantea el régimen excepcional de usos y edificaciones por razones de interés público, conforme a lo previsto en la Ley estatal de suelo y con las condiciones y supuestos señalados en la LOTURM. Para aclarar este régimen excepcional la Consejería de Presidencia y Fomento ha dictado una Instrucción sobre el régimen de autorización excepcional por interés público regulado en la Ley 13/2015, de 30 de marzo, de Ordenación Territorial y Urbanística de la Región de Murcia ${ }^{13}$.

En ella se precisan, siguiendo el régimen de la autorización excepcional de edificación y usos en la legislación del suelo (Real Decreto-Legislativo 7/2015, de 30 de octubre, por el que se aprobó el Texto Refundido de la Ley de suelo y rehabilitación urbana), conceptos tales como el régimen de autorización excepcional por interés público en la LOTURM; la competencia y alcance de la autorización excepcional por interés público prevista en el artículo 104 de la LOTURM; las cuestiones atinentes a la tramitación del procedimiento para esta autorización excepcional; los informes que se consideren necesarios antes de la resolución de la autorización; así como en fin las condiciones, requisitos y documentación que deben acompañar a la solicitud de autorización excepcional.

Otra de las novedades más significativas de la LOTURM ha sido la implementación de los títulos habilitantes de naturaleza urbanística que ha suscitado diversas dudas de interpretación y aplicación en los profesionales y en la administración local, como responsable de la aplicación de dichos instrumentos de control de las actividades objeto de la ordenación territorial y urbanística, por lo que se ha demandado desde diversas instancias una instrucción aclaratoria de determinados aspectos de la ley y con relación a

\footnotetext{
${ }^{13}$ Orden, de 13 de diciembre de 2017, de la Consejería de Presidencia y Fomento, de aprobación de la Instrucción sobre el régimen de autorización excepcional por interés público regulado en la Ley 13/2015, de 30 de marzo, de Ordenación Territorial y Urbanística de la Región de Murcia (BORM, núm. 289, de 16 de diciembre de 2017).
} 
otros instrumentos concurrentes. Así pues, la Consejería de Presidencia y Fomento ha dictado una última Instrucción interpretativa cuyo objeto se centra los títulos habilitantes de naturaleza urbanística regulados en la LOTURM ${ }^{14}$. De acuerdo con esto, y teniendo presente que, pese a que el Estado carece de competencias en materia urbanística, la CE le asigna un papel fundamental en cuanto a la igualdad en el ejercicio de derechos y deberes relacionados con el suelo y las bases económicas y medioambientales de su régimen jurídico, la primera cuestión tratada en la Instrucción se centra en el análisis de las disposiciones reguladoras de los instrumentos de intervención y control de la administración en la actividad urbanística; esto es, en el régimen de las licencias y autorizaciones administrativas en la legislación básica estatal.

En desarrollo de dichas disposiciones básicas, la LOTURM regula bajo la denominación genérica de "títulos habilitantes de naturaleza urbanística", tres modalidades: la licencia urbanística, la declaración responsable y la comunicación previa en materia de urbanismo a los que se hace referencia en la legislación estatal, pero con los condicionantes propios como el referido al establecimiento del silencio administrativo negativo expreso de los actos señalados en el RDL 7/2015 de 30 de octubre, por el que se aprobó el Texto Refundido de la Ley de suelo y rehabilitación urbana. Así pues, la Instrucción analiza detenidamente el régimen y procedimiento que corresponde a cada una de estas tres figuras y su relación con otras autorizaciones y licencias.

\subsection{Residuos}

\section{A. Recogida de residuos en el mar}

En el año 1996 la Consejería de Medio Ambiente, Agricultura y Agua junto con los Ayuntamientos costeros de Águilas, Mazarrón, Cartagena, San Pedro del Pinatar y la Federación Murciana de Cofradías de Pescadores de la Región de Murcia, iniciaron un proyecto cuyo principal objetivo consistía en la retirada del mar de los residuos sólidos aparecidos en las redes de pesca de la flota de arrastre en el ejercicio diario de su actividad, su traslado a puerto y depósito en

\footnotetext{
${ }^{14}$ Orden, de 13 de diciembre de 2017, de la Consejería de Presidencia y Fomento, de aprobación de la Instrucción relativa a los títulos habilitantes de naturaleza urbanística regulados en la Ley 13/2015, de 30 de marzo, de Ordenación Territorial y Urbanística de la Región de Murcia (BORM, núm. 289, de 16 de diciembre de 2017).
} 
contenedores de basura habilitados a tal fin, incluyéndose posteriormente como nueva actuación a desarrollar el suministro de datos obtenidos por las lonjas pesqueras sobre las capturas desembarcadas en los diferentes puertos de la Región de Murcia ${ }^{15}$.

El proyecto se ha venido ejecutando desde entonces mediante la aprobación anual de una subvención por concesión directa a la Federación Murciana de Cofradías de Pescadores, si bien en el año 2016, se canalizó expidiéndose orden de concesión directa individualizada para cada beneficiario tripulante. La peculiar naturaleza de las actuaciones a desarrollar obliga a que sean los propios tripulantes de las embarcaciones de arrastre con puerto base en la Región de Murcia, los que lleven a efecto la recogida de los residuos sólidos a través de las embarcaciones dedicadas a la actividad pesquera dada la imposibilidad de promover concurrencia pública, al ser inviable su ejecución por parte de cualquier otro agente social y además teniendo en cuenta la experiencia satisfactoria acumulada por los mismos en dicho campo de acción desde el año 1996.

De acuerdo con lo anterior, mediante el Decreto 6/2018, de 31 de enero, el Consejo de Gobierno aprueba las normas especiales reguladoras de la subvención a otorgar a través del procedimiento de concesión directa para la recogida de residuos sólidos marinos por parte de los pescadores enrolados en barcos de la modalidad de arrastre con puerto base en la Región de Murcia pertenecientes a las cofradías integradas en la Federación Murciana de Cofradías de Pescadores ${ }^{16}$.

Dado el interés público y social, representado por la mejora de las condiciones ambientales del medio marino, al reducirse sus niveles de contaminación derivados de la intervención humana, ayudando a la consecución de un medio ambiente adecuado para el desarrollo de la persona como aspiración

\footnotetext{
${ }^{15}$ Se define como basura marina a cualquier sólido persistente de origen no natural (manufacturado), que haya sido desechado, depositado o abandonado en ambientes marinos y/o costeros. Esta definición incluye aquellos objetos con origen en las actividades humanas que llegan al medio marino a través de ríos, sistemas de alcantarillado y depuración de aguas o empujados por el viento u otros desde la zona terrestre. Se consideran basuras marinas, materiales tales como: plásticos, madera, metales, vidrio, goma, telas, papel, incluyendo los derivados o desechados de las actividades pesqueras. Esta definición no incluye objetos ni material semisólido o viscoso como aceites vegetales o minerales, petróleo, parafina u otras sustancias químicas contaminantes.

${ }^{16}$ BORM núm. 32, 8 de febrero de 2018.
} 
consagrada en el artículo $45 \mathrm{CE}$, el Decreto autoriza la concesión de una subvención por importe máximo total de 50.000 euros, para el presente ejercicio presupuestario que serán cofinanciados el Fondo Europeo Marítimo y de Pesca, siendo los porcentajes los siguientes: 75 \% FEMP y el restante $25 \%$ de fondos propios afectados.

\section{B. Residuos sólidos}

La Consejería de Turismo, Cultura y Medio Ambiente ha hecho público el contrato programa para el ejercicio 2017, suscrito el pasado 27 de julio con el Consorcio para la Gestión de Residuos Sólidos de la Región de Murcia ${ }^{17}$. Este contrato tiene como misión hacer efectivos los objetivos que, en materia de gestión de residuos sólidos, se establecen en el Plan de Residuos de la Región de Murcia y que fueron examinados en nuestra Crónica anterior ${ }^{18}$.

Tras concretar las responsabilidades asumidas por ambas partes, las obligaciones y derecho del Consorcio para la Gestión de Residuos Sólidos de la Región de Murcia, y la financiación de su ejecución, se especifica la estructura y composición, las áreas operativas, líneas estratégicas y objetivos prioritarios identificados por la Consejería estableciéndose también los plazos para alcanzar estos últimos y los indicadores para su evaluación.

\subsection{Plan de Mejora de la Calidad del Aire para la Región de Murcia 2016- 2018}

El Plan de Mejora de la Calidad del Aire para la Región de Murcia 2016-2018, aprobado por el Consejo de Gobierno el 25 de noviembre de $2015^{19}$, contempla la creación de una Comisión para el seguimiento de su cumplimiento y su revisión, como un órgano colegiado en el que participarán representantes de todas las Administraciones, en especial de los organismos competentes en

\footnotetext{
${ }^{17}$ Resolución de 27 de noviembre de 2017 de la Secretaría General de la Consejería de Turismo, Cultura y Medio Ambiente, por la que se dispone la publicación en el Boletín Oficial de la Región de Murcia del contrato programa para el ejercicio 2017 entre la Consejería de Turismo, Cultura y Medio Ambiente y el Consorcio para la Gestión de Residuos Sólidos de la Región de Murcia (BORM, núm. 284, de 11 de diciembre de 2017).

${ }^{18}$ Revista Catalana de Dret Ambiental, Vol. VIII, Núm. 1 (2017), pp. 1-19.

${ }^{19}$ Plan de Mejora de la Calidad del Aire para la Región de Murcia 2016-2018, hecho público por Resolución, de 20 de enero de 2016, de la Dirección General de Medio Ambiente (BORM, núm. 35, de 12 de febrero de 2016).
} 
materia de Salud Pública, Industria, Transporte, Emergencias, Fomento, Educación y de los Ayuntamientos, así como por agentes sociales y ciudadanos. Creada esta comisión de seguimiento por Orden, de 29 de junio de 2017, fueron nombrando vocales de la misma 36 representantes designados por las entidades públicas o privadas, que aceptaron la invitación de participar $^{20}$.Si bien, con posterioridad a la creación de la Comisión de Seguimiento del Plan de Mejora de la Calidad del Aire para la Región de Murcia 2016-2018, ha habido otras entidades que han manifestado su voluntad de ser incluidas como vocales en la Comisión de Seguimiento, motivo por el que para asegurar una mayor difusión de la información sobre calidad del aire y garantizar que se halle a disposición del mayor número posible de ciudadanos, la Dirección General de Medio Ambiente propone modificar la citada Orden incluyendo como vocales en la Comisión de Seguimiento, seis nuevos representantes designados por las entidades públicas o privadas.

De este modo, mediante Orden, de 11 de diciembre de 2017, pasan a integran la comisión de seguimiento los siguientes representantes: un representante designado por Ecologistas en Acción Región de Murcia; un representante designado por la Asociación de Naturalistas del Sureste (ANSE); un representante designado por la Coordinadora de Organizaciones de Agricultores y Ganaderos-Iniciativa Rural de Murcia (COAG-IR); un representante designado por el Partido Popular Región de Murcia; un representante designado por Ciudadanos Región de Murcia y un representante designado por Podemos Región de Murcia ${ }^{21}$.

\subsection{Programa de Medio Ambiente y Acción por el Clima (LIFE)}

Con fecha de 11 de diciembre de 2013 se adoptó el Reglamento (UE) núm. 1.293/2013 del Parlamento Europeo y del Consejo, por el que se establece el Programa de Medio Ambiente y Acción por el Clima (LIFE) que abarca el

\footnotetext{
${ }^{20}$ Orden, de 29 de junio de 2017 de la Consejería de Turismo, Cultura y Medio Ambiente, por la que se crea la Comisión de Seguimiento del Plan de Mejora de la Calidad del Aire para la Región de Murcia 2016-2018 (BORM, núm. 165, de 19 de julio de 2017).

${ }^{21}$ Orden de 11 de diciembre de 2017, de la Consejería de Turismo, Cultura y Medio Ambiente, de modificación de la Orden de 29 de junio de 2017 por la que se crea la Comisión de Seguimiento del Plan de Mejora de la Calidad del Aire para la Región de Murcia 2016-2018, (BORM núm. 288, de 15 de diciembre de 2017).
} 
período comprendido entre el 1 de enero de 2014 y el 31 de diciembre de 2020. Su objetivo general se basa en catalizar los cambios en el desarrollo y la aplicación de las políticas mediante la aportación de soluciones y mejores prácticas para lograr los objetivos medioambientales y climáticos, así como mediante la promoción de tecnologías innovadoras en materia de medio ambiente y cambio climático.

La Oficina de Impulso Socioeconómico del Medio Ambiente (OISMA), dependiente de la Dirección General de Medio Natural de la Consejería de Turismo, Cultura y Medio Ambiente, ha sido y es beneficiaria y coordinadora de varios Acuerdos de Subvención europeos, a través del instrumento financiero "Programas LIFE", con la posibilidad de la suscripción de nuevos acuerdos hasta el año $2020^{22}$. Para la ejecución de los proyectos LIFE que así lo prevean, los empleados públicos vinculados al proyecto podrán contar con la participación de personal subvencionado (becarios) para la realización de acciones concretas en el marco de cada uno de los proyectos, mediante la concesión de becas que tienen por finalidad la promoción de la formación y el perfeccionamiento técnico-profesional en la gestión de los programas LIFE. De este modo, la Consejería de Turismo, Cultura y Medio Ambiente, establece mediante la Orden, de 12 de febrero de 2018 las bases reguladoras para la solicitud y concesión de estas becas, al objeto de efectuar la asignación de las mismas en las condiciones de publicidad, concurrencia, objetividad, transparencia, igualdad y no discriminación, eficacia en el cumplimiento de los objetivos que se establezcan ${ }^{23}$.

\footnotetext{
${ }^{22}$ Según el Decreto del presidente 3/2017, de 4 de mayo, de reorganización de la Administración Regional, la Consejería de Turismo, Cultura y Medio Ambiente es el Departamento de la Comunidad Autónoma de la Región de Murcia encargado de la propuesta, desarrollo y ejecución de las directrices generales del Consejo de Gobierno en materia de medio ambiente. Según Decreto 75/2017, de 17 de mayo, por el que se establecen los Órganos Directivos de la Consejería de Turismo, Cultura y Medio Ambiente (modificado por Decreto 223/2017, de 2 de agosto), la Dirección General de Medio Natural, en la que se integra la Oficina de Impulso Socioeconómico del Medio Ambiente con rango de Subdirección General, asume las competencias y funciones en materia de planificación y gestión de espacios naturales protegidos de la Red Natura 2000, de los hábitats naturales y de la fauna y flora silvestres, así como del fomento del medio ambiente y lucha contra el cambio climático, la representación en la Red de Autoridades Ambientales, política forestal, caza y pesca fluvial y protección de la fauna silvestre.

${ }^{23}$ Orden, de 12 de febrero de 2018 de la Consejería de Turismo, Cultura y Medio Ambiente por la que se aprueban las bases reguladoras para la concesión de becas en el marco de programas LIFE de la Unión Europea (Programas de Medio Ambiente y Acción por el Clima), (BORM, núm. 58, de 10 de marzo de 2018).
} 


\subsection{Premios de desarrollo sostenible}

Los premios en materia de medio ambiente tienen en la Región de Murcia un largo recorrido, convocándose por primera vez en el año 2002 con el nombre de Premios de Calidad Ambiental. En 2006, y recogiendo las recomendaciones contenidas en el Pacto Social por el Medio Ambiente, pasaron a denominarse Premios de Desarrollo Sostenible ${ }^{24}$. El objetivo de estos premios de desarrollo sostenible es reconocer públicamente el esfuerzo realizado por empresas, instituciones, organizaciones y colectivos de la Región en materia de desarrollo sostenible, y para difundir aquellas experiencias de éxito e ideas que puedan ser referente para otros. En atención a esto, la Consejería de Agua, Agricultura y Medio Ambiente, mediante la Orden, de 25 de abril de 2017, convocó la novena edición de los Premios de Desarrollo Sostenible de la Región de Murcia, resuelta por la Orden, de 29 de diciembre de 2017, de la Consejería de Turismo, Cultura y Medio Ambiente, por la que se conceden los galardones ${ }^{25}$.

\subsection{Agricultura}

\section{A. Medidas fitosanitarias}

Mediante la Orden, de 19 de octubre de 2017, de la Consejería de Agua, Agricultura, Ganadería y Pesca, se dictan las medidas fitosanitarias a adoptar en caso de acumulación de restos vegetales procediendo en su artículo 1 a declarar la quema controlada de restos vegetales procedentes de la poda u otras operaciones de cultivo generados en la propia explotación, como medida fitosanitaria para evitar la propagación de plagas, reducir su población, mitigar sus efectos, o conseguir su erradicación de los organismos nocivos de determinados cultivos previstos en dicho artículo ${ }^{26}$.

Entre los cultivos incluidos en dicho artículo no se ha tenido en cuenta el cultivo del arroz. Sin embargo, considerando que la quema de este cultivo, realizada

\footnotetext{
${ }^{24} C f r$. Protocolo General suscrito entre la Consejería de Industria y Medio Ambiente y las organizaciones representativas de la actividad económica y social de la Región de Murcia para fomentar el desarrollo sostenible en el marco del Pacto Social por el Medio Ambiente, autorizado por el Consejo de Gobierno en sesión de 17 de noviembre de 2006 (BORM, núm. 20, de 25 de enero de 2007).

${ }^{25}$ Orden, de 29 de diciembre de 2017, de la Consejería de Turismo, Cultura y Medio Ambiente, por la que se conceden los Premios de Desarrollo Sostenible correspondientes a su novena edición (BORM, núm. 8, de 11 de enero de 2018).

${ }^{26}$ BORM, núm. 244, de 21 octubre de 2017.
} 
bajo condiciones controladas y en combinación con otras operaciones de labor y mantenimiento del suelo, puede contribuir eficientemente al control de los daños ocasionados por anoxia o akiochi, así como la lucha contra la Pedicularia orizae y Chilo supresalis (barrenador del arroz) e, indirectamente, reducir los niveles de población de vectores y formas latentes de plagas y enfermedades que deberían combatirse en el siguiente ciclo de cultivo con productos fitosanitarios, resulta necesaria la modificación de la Orden, a fin de regular que la quema controlada de los restos de cultivo de arroz se encuentre incluida también como medida fitosanitaria para evitar la propagación de plagas y enfermedades.

En consecuencia, la Consejería de Agua, Agricultura, Ganadería y Pesca mediante Orden, de 6 de noviembre de 2017, de la Consejería de Agua, Agricultura, Ganadería y Pesca, acuerda la modificación de la Orden, de 19 de octubre de 2017, por la que se dictan las medidas fitosanitarias a adoptar en caso de acumulación de restos vegetales, dando una nueva redacción a su artículo 1 incluyendo el arroz entre los cultivos cuyos restos vegetales, procedentes de la poda $u$ otras operaciones de cultivo generados en la propia explotación, deben sometidos a que controlada como medida fitosanitaria ${ }^{27}$.

\section{B. Agricultura ecológica y denominación de origen}

Mediante Orden, de 1 de diciembre de 2017, la Consejería de Agua, Agricultura, Ganadería y Pesca, aprueba la convocatoria para el año 2017 de las ayudas destinadas a los consejos reguladores de las denominaciones de origen, indicaciones geográficas protegidas y Consejo de Agricultura Ecológica para la realización de sus actividades ${ }^{28}$. En concreto, estas ayudas tienen como finalidad contribuir a las actuaciones relacionadas con la implantación y desarrollo de programas de mejora y control de la calidad y del origen de los

\footnotetext{
${ }^{27}$ Orden, de 6 de noviembre de 2017, de la Consejería de Agua, Agricultura, Ganadería y Pesca, por la que se modifica la Orden, de 19 de octubre de 2017, por la que se dictan las medidas fitosanitarias a adoptar en caso de acumulación de restos vegetales (BORM, núm. 259, de 9 de noviembre de 2017).

${ }^{28}$ Orden, de 1 de diciembre de 2017 de la Consejería de Agua, Agricultura, Ganadería y Pesca, por la que se aprueba la convocatoria para el año 2017 de las ayudas destinadas a los consejos reguladores de las denominaciones de origen, indicaciones geográficas protegidas y Consejo de Agricultura Ecológica para la realización de sus actividades (BORM, núm. 280, de 4 de diciembre de 2017).

Las bases para estas convocatorias fueron se hicieron públicas mediante Orden, de 17 de julio de 2017 (BORM, núm. 167, de 21 de julio de 2017), modificada por Orden, de 14 de noviembre de 2017 (BORM, núm. 266, de 17 de noviembre de 2017).
} 
productos agroalimentarios murcianos amparados por alguna denominación geográfica de calidad con sede en la Región de Murcia o acogidos a la producción ecológica. El importe total de la convocatoria es de $300.000 €$ que deberán ser destinados a los gastos subvencionables, esto es:

a) Las actuaciones de control realizadas, directa o indirectamente, por los consejos reguladores de las denominaciones de origen, indicaciones geográficas protegidas y Consejo de Agricultura Ecológica encaminadas a garantizar la calidad y el origen de los productos de la correspondiente denominación.

b) Costes derivados de las actividades de promoción e información soportados por los consejos reguladores de las denominaciones de origen, indicaciones geográficas protegidas y Consejo de Agricultura Ecológica. Estas ayudas tienen como objeto la promoción de los productos agroalimentarios.

C. Programa de Desarrollo Rural de la Región de Murcia 2014-2020: ayudas para las medidas de "agroambiente y clima" y "agricultura ecológica"

Como tuvimos ocasión de señalar en crónicas anteriores, a finales del año 2013 se inició en la Unión Europea el nuevo período de programación de desarrollo rural 2014-2020, cuyo marco normativo está constituido por un conjunto de disposiciones comunitarias a cuyo frente se sitúa el Reglamento núm. 1305/2013, del Parlamento Europeo y del Consejo, de 17 de diciembre de 2013, relativo a la ayuda al desarrollo rural a través del Fondo Europeo Agrícola de Desarrollo Rural (FEADER) ${ }^{29}$. Este Reglamento estructura las distintas medidas de desarrollo rural que pueden cofinanciarse a través del mencionado Fondo, entre las que destacan las destinadas al agroambiente y clima y a la agricultura ecológica.

De acuerdo con esto, la Consejería de Agricultura y Agua propuso a la Comisión Europea el Programa de Desarrollo Rural de la Región de Murcia, en el que se incluyeron medidas relativas al "agroambiente y clima" y a la “agricultura ecológica".

\footnotetext{
${ }^{29}$ Revista Catalana de Dret Ambiental, Vol. VI, Núm. 2, 2015, p. 1-15.
} 
Mediante la Orden, de 25 de mayo de 2015, de la Consejería de Agricultura y Agua, se establecieron las bases y las convocatorias para las líneas de ayuda incluidas en las medidas 10 y 11 del Programa de Desarrollo Rural de la Región de Murcia $^{30}$. Una vez concedidas las ayudas, a la vista de los créditos disponibles procedentes del Programa de Desarrollo Rural y teniendo en cuenta tanto la experiencia adquirida durante la concesión de las ayudas, como las demandas de los agricultores y la necesidad de incrementar la protección de los ecosistemas agrarios, es necesario proceder a modificar algunos aspectos de las bases reguladoras de las ayudas y a tal fin, la Consejería de Agua, Agricultura Ganadería y Pesca dicta la Orden, de 16 febrero de 2018, entre cuyas medidas destaca la fijación de las primas de la línea de ayuda a lucha biotecnológica para las anualidades 2017-2020 para cada uno de los cultivos subvencionables ${ }^{31}$.

D. Programa de Desarrollo Rural de la Región de Murcia 2014-2020: Ayudas LEADER

El pasado 6 de octubre de 2017 se hicieron públicas las Bases Reguladoras de las Ayudas LEADER previstas en la submedida 19.2 del Programa de Desarrollo Rural de la Región de Murcia 2014-2020, para proyectos no programados en las Estrategias de Desarrollo Local Participativo ${ }^{32}$. La experiencia en la gestión de estas ayudas pone de manifiesto la necesidad de modificar algunos artículos de la Orden, de 3 de octubre de 2017, de la Consejería de Agua, Agricultura, Ganadería y Pesca, por la que se establecen las bases reguladoras de las ayudas LEADER previstas en la submedida 19.2

\footnotetext{
${ }^{30}$ Orden de la Consejería de Agricultura y Agua, de 25 de mayo de 2015, por la que se establecen en la Región de Murcia, las bases reguladoras de determinadas líneas de ayuda correspondientes a la medida 10 "Agroambiente y clima" y medida 11 "Agricultura ecológica" del Programa de Desarrollo Rural de la Región de Murcia 2014-2020, y se aprueba la convocatoria correspondiente al año 2015 de las citadas líneas de ayuda (BORM, núm. 120, de 27 de mayo de 2015).

${ }^{31}$ Orden, de 16 febrero de 2018, por la que se modifica la Orden, de 25 de mayo de 2015, de la Consejería de Agricultura y Agua, por la que se establecen, en la Región de Murcia, las bases reguladoras de determinadas líneas de ayuda correspondientes a la medida 10 "Agroambiente y Clima" y medida 11 "Agricultura Ecológica" del programa de desarrollo rural de la Región de Murcia 2014-2020, y se aprueba la convocatoria correspondiente al año 2015 de las citadas líneas de ayuda (BORM, núm. 45, de 23 de febrero de 2018).

${ }^{32}$ Orden, de 3 de octubre de 2017, por la que se establecen las Bases Reguladoras de las ayudas LEADER previstas en la submedida 19.2 del Programa de Desarrollo Rural de la Región de Murcia 2014-2020, para proyectos no programados en las Estrategias de Desarrollo Local Participativo (BORM, núm. 232, de 6 de octubre de 2017).
} 
del Programa de Desarrollo Rural de la Región de Murcia 2014-2020, para proyectos no programados en las Estrategias de Desarrollo Local Participativo. Así pues, mediante la Orden, de 9 de noviembre de 2017, se modifica la Orden anterior ${ }^{33}$.

En concreto, la modificación afecta a los beneficiarios de las ayudas, a los controles administrativos de todas las solicitudes de ayuda, a las solicitudes de pago u otras declaraciones que presenten o deban presentar los beneficiarios, a la moderación de los costes en las inversiones o actuaciones para las que se solicita ayuda, al proceso de aprobación de la convocatoria de proyectos no programados de la submedida 19.2, al inicio, instrucción y resolución del procedimiento de concesión de las ayudas.

\subsection{Pesca}

A. Veda temporal para el ejercicio de la pesca de cerco en aguas interiores de la Región de Murcia

La Política Pesquera Comunitaria de la Unión Europea tiene entre sus objetivos el asegurar que la explotación de los recursos biológicos marinos vivos se orienta al mantenimiento de las poblaciones de especies capturadas, por encima del nivel de rendimiento máximo sostenible ${ }^{34}$. Para la consecución de dicha finalidad, la actuación de la Administración pública de la Región de Murcia se dirige a lograr una explotación equilibrada y responsable de los recursos pesqueros, así como concerniente a la pesca recreativa, a la promoción del ejercicio responsable de la misma.

Así pues, la Consejería de Agua, Agricultura, Ganadería y Pesca, con el fin de facilitar la consecución de los objetivos biológicos y de gestión sostenible de las

\footnotetext{
${ }^{33}$ Orden, de 9 de noviembre de 2017, por la que se modifica la Orden, de 3 de octubre de 2017 de la Consejería de Agua, Agricultura, Ganadería y Pesca, por la que se establecen las bases reguladoras de las ayudas LEADER previstas en la submedida 19.2 del Programa de Desarrollo Rural de la Región de Murcia 2014-2020, para proyectos no programados en las Estrategias de Desarrollo Local Participativo (BORM, núm. 261, de 11 de noviembre de 2017).

${ }^{34}$ El Reglamento (CE) 1.967/2006 del Consejo, de 21 de diciembre de 2006, relativo a las medidas de gestión para la explotación sostenible de los recursos pesqueros en el Mar Mediterráneo, marca como objetivo principal establecer un marco de gestión eficaz para la protección estricta de determinadas especies marinas, así como la conservación de los hábitats naturales y la fauna y flora silvestres. De acuerdo con este, y mediante Orden AAA/2808/2012, de 21 de diciembre, se aprobó el Plan de Gestión Integral para la conservación de los recursos pesqueros en el Mediterráneo afectados por las pesquerías realizadas con artes de cerco, redes de arrastre y artes fijos y menores, para el periodo 2013-2017.
} 
actividades pesqueras realizadas con artes de cerco, redes de arrastre y artes fijos y menores en el caladero del Mediterráneo, ha venido adoptando medidas de limitación del esfuerzo pesquero a través del establecimiento de periodos temporales de veda. Estas medidas se entienden necesarias en el presente periodo motivo por el que la Consejería de Agua, Agricultura, Ganadería y Pesca declara una veda temporal para el ejercicio de la pesca en la modalidad de cerco en aguas interiores de la Región de Murcia desde el día 6 de diciembre de 2017 hasta el día 7 de enero de 2018, ambos inclusive ${ }^{35}$. Asimismo, y respecto al ejercicio de la pesca de arrastre la Consejería declara una veda temporal para el ejercicio de la pesca en la modalidad de arrastre de fondo en aguas interiores de la Región de Murcia desde el día 12 de mayo hasta el día 10 de junio de 2018, ambos inclusive, bajo pena de sanción ${ }^{36}$.

Del mismo modo, el pulpo común (Octopus vulgaris) se encuentra entre las especies autorizadas para su captura en el ejercicio de la pesca marítima de recreo, en todas sus modalidades, dentro del respeto a la normativa vigente en relación a tallas y pesos mínimos permitidos, así como observando los periodos de veda aprobados ${ }^{37}$. Con el fin de garantizar una pesca recreativa sostenible del pulpo en aguas interiores de la Región de Murcia, no interfiriendo ni restando efectividad a las medidas de conservación, protección y regeneración de las poblaciones de pulpo común puestas en marcha para la flota de artes menores $^{38}$, la Consejería de Agua, Agricultura, Ganadería y Pesca dicta la Orden, de 9 de noviembre de 2017 cuyo objeto es establecer una veda estacional para la pesca del pulpo (Octopus vulgaris) afectante a la pesca

\footnotetext{
${ }^{35}$ Haciendo uso de las competencias conferidas por el art. 5 de la Ley 2/2007, de 12 de marzo, de Pesca Marítima y Acuicultura de la Región de Murcia, la Consejería de Agua, Agricultura, Ganadería y Pesca ha dictado la Orden, de 27 de octubre de 2017, por la que se establece un periodo de veda temporal para el ejercicio de la pesca de cerco en aguas interiores de la Región de Murcia (BORM, núm.253, de 2 de noviembre de 2017).

${ }^{36}$ Orden, de 16 febrero de 2018, por la que se establece un periodo de veda temporal para el ejercicio de la pesca de arrastre en aguas interiores de la Región de Murcia (BORM núm. 50, de 1 de marzo de 2018). El ejercicio de la actividad pesquera en dicha modalidad durante el periodo de veda establecido, será sancionado de conformidad con lo dispuesto en la Ley 2/2007, de 12 de marzo, de Pesca Marítima y Acuicultura de la Región de Murcia.

${ }^{37}$ Decreto 72/2016, de 20 de julio, regulador de la pesca marítima de recreo en las aguas interiores de la Región de Murcia (BORM, núm. 170, de 23 de julio de 2016).

${ }^{38}$ Orden, de 30 de abril de 2014 de la Consejería de Agricultura y Agua, regula la pesca artesanal del pulpo (Octopus vulgaris) en aguas de la Comunidad Autónoma de la Región de Murcia, estableciendo dos periodos de veda para la captura de esta especie comprendidos el primero entre el 1 de mayo y el 30 de junio y el segundo entre el 1 de septiembre y el 31 de octubre.
} 
marítima de recreo en aguas interiores de la Región de Murcia $^{39}$. Los periodos de veda anual para la pesca marítima de recreo del pulpo irán del 1 de mayo al 30 de junio (ambos inclusive) y del 1 de septiembre al 31 de octubre (ambos inclusive). Lo dispuesto en dicha orden resulta de aplicación a todas las modalidades de pesca marítima recreativa, tanto a la pesca recreativa de superficie (desde tierra o desde embarcación) como a la pesca submarina (realizada nadando o buceando a pulmón libre).

\section{B. Reserva Marina zona de Cabo de Palos-Islas Hormigas}

Desde la declaración de la zona de cabo de Palos-Islas Hormigas como reserva marina por Decreto 15/1995, de 31 de marzo (en aguas interiores) y Orden Ministerial, de 22 de junio de 1995 (en aguas exteriores), la Consejería de Agua, Agricultura y Medio Ambiente viene realizando diversos trabajos de seguimiento de los efectos derivados de su declaración, en especial sobre las poblaciones de especies piscícolas de interés comercial mediante el proyecto "Seguimiento de la Reserva Marina Cabo de Palos-Islas Hormigas 2016".

Prueba de ello son el Convenio de colaboración, suscrito el 14 de diciembre de 2001, entre el Ministerio de Agricultura, Pesca y Alimentación y la Consejería de Agricultura, Agua y Medio Ambiente, relativo a la gestión compartida de la reserva marina del entorno de Cabo de Palos-Islas Hormigas ${ }^{40}$, y los convenios que, desde el 2003, se han suscrito entre la Consejería de Agricultura, Agua y Medio Ambiente y la Universidad de Murcia para la realización conjunta de trabajos de seguimiento de esta zona ${ }^{41}$. En esta línea, mediante Decreto 42/2015, de 27 de marzo, se otorgó, por concesión directa, una subvención a la Universidad de Murcia para la continuación de los citados trabajos en el marco

\footnotetext{
${ }^{39}$ Orden, de 9 de noviembre de 2017 de Consejería de Agua, Agricultura, Ganadería y Pesca por la que se establece una veda para la pesca marítima de recreo del pulpo (Octopus vulgaris) en aguas interiores del litoral de la Comunidad Autónoma de la Región de Murcia (BORM, núm. 263, de 14 de noviembre de 2017).

${ }^{40}$ Convenio de colaboración entre el Ministerio de Agricultura, Pesca y Alimentación y la Consejería de Agricultura, Agua y Medio Ambiente relativo a la gestión compartida de la reserva marina del entorno de Cabo de Palos-Islas Hormigas (BORM, núm. 29, de 4 de febrero de 2002), prorrogado hasta el 11 de julio de 2018 por Acuerdo de prórroga del convenio de colaboración entre ambas administraciones (BORM, núm. 253, de 31 de octubre de 2013).

${ }^{41}$ Convenios de colaboración de 2003, 2006-2007, 2008, 2009, 2010, 2012-2013 y 2014-2015, publicados en el BORM de 18 de septiembre de 2003, 4 de agosto de 2006, 24 de julio de 2008, 29 de julio de 2009, 29 de julio de 2010, 14 de noviembre de 2012 y 22 de diciembre de 2014, respectivamente.
} 
del proyecto "Seguimiento de la Reserva Marina Cabo de Palos-Islas Hormigas $2016 " 42$.

Los resultados obtenidos han servido de base para mejorar la gestión de la reserva marina y para diseñar las actuaciones relacionadas con su uso público - tanto para regular el flujo de visitantes como el sector pesquero que faena en la zona- por lo que se ha considerado plenamente justificado el interés público de la ayuda. En el presente año estos estudios se van a financiar de nuevo con cargo al Fondo Europeo Marítimo y de Pesca, financiación que se fundamenta en la aplicación del artículo 28 del Reglamento 508/2014, regulador del Fondo Europeo Marítimo y de Pesca, sobre el fomento de un sector pesquero innovador basado en el conocimiento científico-técnico.

A la vista de la naturaleza del trabajo a realizar y teniendo en cuenta la experiencia en este campo del Grupo de Investigación de Ecología y Conservación Marina del Departamento de Ecología e Hidrología de la Universidad de Murcia en este campo, así como del Área de Pesca del Instituto Español de Oceanografía, se considera de gran interés que la Comunidad Autónoma mantenga su participación en el proyecto financiándolo mediante la concesión de una subvención directa a ambas entidades, a fin de que sean éstas quienes continúen con los trabajos de seguimiento que se han venido llevando a cabo en las reservas marinas.

A tal fin, el Decreto 20/2018, de 14 de marzo, del Consejo de Gobierno, aprueba las normas especiales que regulan la subvención a otorgar mediante concesión directa por la Consejería de Agua, Agricultura, Ganadería y Pesca a la Universidad de Murcia y al Instituto Español de Oceanografía para continuar con el desarrollo de un proyecto de investigación sobre los efectos de la declaración como reservas marinas de las zonas de Cabo de Palos-Islas Hormigas y de Cabo Tiñoso ${ }^{43}$.

\footnotetext{
42 Decreto 42/2015, de 27 de marzo, por el que se aprueban las normas especiales reguladoras de una subvención a otorgar por concesión directa a la Universidad de Murcia para continuar con la ejecución del proyecto de investigación sobre los efectos de la reserva marina de Cabo de Palos-Islas Hormigas (BORM, núm. 74, de 31 de marzo de 2015).

43 Decreto 20/2018, de 14 de marzo, del Consejo de Gobierno, por el que se aprueban las normas especiales reguladoras de una subvención a otorgar mediante concesión directa por la Consejería de Agua, Agricultura, Ganadería y Pesca a la Universidad de Murcia y al Instituto Español de Oceanografía para continuar con el desarrollo de un proyecto de investigación sobre los efectos de la declaración como
} 


\section{ORDENANZAS LOCALES}

\subsection{Murcia}

A. Aprobación inicial de la ordenanza municipal sobre protección y tenencia de animales de convivencia humana ${ }^{44}$

El Pleno de este Ayuntamiento, en sesión celebrada el 26 de octubre de 2017 aprobó inicialmente la ordenanza municipal sobre protección y tenencia de animales de convivencia humana. En consecuencia, se abre un período de información pública para reclamaciones y sugerencias por plazo de treinta días, según lo preceptuado en los artículos 49 y 70.2 de la Ley $7 / 1985$, durante el cual podrá ser examinado el expediente en las dependencias de las Servicios Municipales de Salud y presentar las reclamaciones o sugerencias que se estimen convenientes.

B. Aprobación inicial del Plan Especial de Ordenación y Protección de la Zona Arqueológica del Arrabal de la Arrixaca Nueva ${ }^{45}$

Aprobado inicialmente por la Junta de Gobierno del Excmo. Ayuntamiento de Murcia, en sesión celebrada el día 22 de diciembre de 2017, el "Plan Especial de ordenación y Protección de la Zona Arqueológica del Arrabal de la Arrixaca Nueva", junto con el documento ambiental que incorpora, se somete a información pública, de conformidad con los artículos 155 y 164, letra b, de la ley $13 / 2015$, de 30 de marzo, de Ordenación Territorial y Urbanística de la Región de Murcia, por plazo de 1 mes, para la presentación de alegaciones.

\subsection{Caravaca de la Cruz: aprobación inicial de la ordenanza municipal para la protección y uso sostenible del paraje de Las Fuentes del Marqués ${ }^{46}$.}

El Ayuntamiento Pleno, en sesión ordinaria celebrada el día 30 de octubre de 2017, aprobó inicialmente la ordenanza municipal para la protección y uso

\footnotetext{
reservas marinas de las zonas de Cabo de Palos-Islas Hormigas y de Cabo Tiñoso (BORM, núm. 64, de 17 de marzo de 2018).

${ }^{44}$ BORM, núm. 259, de 9 de noviembre de 2017.

${ }^{45}$ BORM, núm. 15, de 19 de enero de 2018

${ }^{46}$ BORM, núm. 271, 23 de noviembre de 2017.
} 
sostenible del paraje de las Fuentes del Marqués. Se abre un plazo de treinta días a efectos de reclamaciones transcurridos los cuales se considerará definitivamente aprobada publicándose la misma íntegramente en el Boletín Oficial de la Región de Murcia.

3.3. Molina de segura: aprobación inicial de la modificación de la ordenanza municipal para la tenencia, protección y bienestar de los animales en Molina de Segura ${ }^{47}$.

El Pleno del Ayuntamiento de Molina de Segura, en la sesión ordinaria celebrada el 30 de octubre de 2017, ha aprobado con carácter inicial la "modificación de la ordenanza municipal para la tenencia, protección y bienestar de los animales en Molina de Segura". Se acuerda abrir un periodo de información pública y audiencia por un plazo de treinta días hábiles, durante el que los interesados pueden examinar el texto completo de la Ordenanza y presentar las reclamaciones y sugerencias que estimen oportunas que, de producirse, deberán ser resueltas por el Pleno de la Corporación.

En caso de que no se presentasen reclamaciones o sugerencias en el indicado plazo, se entenderá definitivamente aprobada la modificación de la Ordenanza, procediéndose a su publicación en el Boletín Oficial de la Región de Murcia, a los efectos de su entrada en vigor.

\subsection{Los Alcázares: Reglamento de autorización del procedimiento de} otorgamiento de autorizaciones de dominio público y del régimen jurídico de los Huertos Urbanos del Ayuntamiento de Los Alcázares ${ }^{48}$

En la sesión ordinaria del 3 de octubre de 2017, el Pleno de la corporación de Los Alcázares acordó someter a información pública el Reglamento Regulador del Procedimiento de Otorgamiento de Autorizaciones de Dominio Público y del Régimen Jurídico de los Huertos Urbanos del Ayuntamiento por el que se pretende organizar una actividad lúdico-educativa enfocada a los ciudadanos del municipio con el fin de fomentar la participación de todos los sectores de la población, con los siguientes objetivos: i) recuperar y conservar una agricultura

\footnotetext{
${ }^{47}$ BORM, núm. 24, de 30 de enero de 2018.

${ }^{48}$ BORM, núm. 25, de 31 de enero de 2018.
} 
tradicional; ii) inculcar una agricultura respetuosa con el medio ambiente, cultural, sostenible: agricultura ecológica; iii) fomentar la adquisición de una serie de valores que motiven a sentir interés y preocupación por conservar la biodiversidad y los hábitats naturales de las especies hortícolas tradicionales y autóctonas; iv) fomentar la participación ciudadana; v) establecer y valorar las relaciones entre el medio natural y las actividades humanas; vi) facilitar el contacto directo y la manipulación de elementos como la tierra, el agua, las plantas; vii) proporcionar al colectivo de mayores una actividad lúdicoeducativa, donde además de las labores propias de la actividad, podrán enriquecer con consejos propios de su experiencia; viii) igualmente, el presente Reglamento contiene el régimen jurídico al que se someterán las personas autorizadas, estableciendo unas normas de funcionamiento de dichos huertos urbanos.

De este modo, transcurrido el tiempo legalmente establecido para la exposición pública sin que se hayan presentado reclamaciones, se acuerda elevar a definitivo el acuerdo por el que se establecen las condiciones técnicas y jurídicas para la concesión de autorizaciones de uso de los 25 Huertos Urbanos —con una superficie de 49,69 $\mathrm{m}^{2}$ — situados en la parcela ubicada en la Urbanización Oasis del municipio de Los Alcázares. 